\title{
Improved Interferometric Method to Measure Near-Carrier AM and PM Noise
}

\author{
Enrico Rubiola, Vincent Giordano, and Jacques Groslambert
}

\begin{abstract}
An improved version of the interferometric method to measure near-carrier AM and PM noise is being presented. The main feature of this scheme is the capability to reduce the instrument noise by correlating the output of two equal interferometers built around the same device to be tested, thus enhancing the sensitivity. Two double interferometers are described, operating in the microwave and VHF bands. The latter shows a noise floor of $-194 \mathrm{~dB} \mathrm{rad}^{2} / \mathrm{Hz}$ when the signal power is $+8 \mathrm{dBm}$. The sensitivity of both the instruments turns out to be significantly higher than the ratio of the thermal noise divided by the carrier power.
\end{abstract}

Index Terms - AM noise, correlation, phase noise, random noise.

\section{INTRODUCTION}

$\mathbf{I}$ T HAS been recognized recently that the interferometric method, although based on old ideas [1], allows real-time near-carrier noise measurement in the microwave region with higher sensitivity compared to the traditional method based on a saturated mixer. In fact, it has been demonstrated that for the X-band a PM noise measurement system (NMS), based on this method, shows a sensitivity $S_{\varphi 0}(f)$ close to $-190 \mathrm{~dB}$ $\mathrm{rad}^{2} / \mathrm{Hz}$ (white noise) if a power level as high as $20 \mathrm{dBm}$ can be tolerated [2]. Besides, it is known that the sensitivity of the saturated-mixer NMS can be improved by some $15 \mathrm{~dB}$ by means of correlation of the output of two equal NMS's that measure the same device [3], [11]. So, in search of the most sensitive instrument we combined the two techniques, correlating the output of two interferometers measuring the same DUT (device under test). After having proposed some preliminary results [4], [5], full details and experiments are being presented. Two NMS's were built, operating at $7.3 \mathrm{GHz}$ and $100 \mathrm{MHz}$, with sensitivity values of $-192 \mathrm{~dB} \mathrm{rad}^{2} / \mathrm{Hz}$ and $-194 \mathrm{~dB} \mathrm{rad}^{2} / \mathrm{Hz}$ with carrier power of 8 and $15 \mathrm{dBm}$, respectively.

As the noise theory of the proposed NMS is still under development, we will spend most of the following sections describing the instrument and the experiments.

\section{BASICS OF THE DOUBLE INTERFEROMETER}

The scheme of the double interferometer is shown in Fig. 1. Let us first analyze one of the two interferometers, for instance that shown in the upper half of the figure. The $90^{\circ}$ hybrid

Manuscript received July 2, 1998.

E. Rubiola is with Politecnico di Torino, 10129 Torino, Italy (e-mail: rubiola@polito.it).

V. Giordano and J. Groslambert are with LPMO CNRS, 25044 Besançon, France (e-mail: giordano@1pmo.univ-fcomte.fr).

Publisher Item Identifier S 0018-9456(99)02902-2. coupler makes the vector addition, i.e., the interference, of its input signals. Thus, setting $\ell^{\prime}$ and $\gamma^{\prime}$ equal to the DUT attenuation and phase shift, respectively, all the carrier power goes to the $\Sigma$ output, and the carrier is suppressed at the $\Delta$ output. The DUT noise sidebands, which are not suppressed by the interference mechanism, are amplified by the low noise amplifier (LNA) and down-converted to baseband by the mixer. The latter down converts PM or AM noise, depending on the detection phase $\gamma^{\prime \prime}$. Setting $\gamma^{\prime \prime}=90^{\circ}$, the voltage $x(t)$ at the output of the mixer is proportional to the instant value $\varphi(t)$ of the phase noise. The power gain $K_{\varphi}$ of the NMS, defined as $K_{\varphi}=x^{2}(t) / \varphi^{2}(t)$, is given by

$$
K_{\varphi}=\frac{R_{0} g_{a} P_{o}}{2 \ell_{s} \ell_{a} \ell_{h} \ell_{m}}
$$

where

$R_{0} \quad$ output impedance of the mixer;

$g_{a} \quad$ power gain of the amplifier;

$P_{o} \quad$ power available at the DUT output;

$\ell_{s} \quad$ loss of the power splitter;

$\ell_{a} \quad$ additional loss needed to compensate the residual loss of the variable attenuator and phase shifter;

$\ell_{h} \quad$ loss of the hybrid;

$\ell_{m} \quad$ loss of the mixer.

According to the usual definitions, $\ell_{s}$ and $\ell_{h}$ do not include the $3 \mathrm{~dB}$ intrinsic loss due to power splitting; $\ell_{m}$, by contrast, includes the $3 \mathrm{~dB}$ intrinsic loss due to the fact that the mixer makes the sum and the difference of its input frequencies, and consequently, it splits the input power into two bands.

Replacing the DUT with a short cable, the residual PSD (power spectrum density) $S_{\varphi 0}(f)$ of the white noise represents the sensitivity of the interferometer, given by

$$
S_{\varphi 0}(f)=\frac{4 F_{a} k_{B} T_{0} \ell_{s} \ell_{h} \ell_{a}}{P_{o}}
$$

where $F_{a}$ is the noise figure of the amplifier and $k_{B}=$ $1.38 \times 10^{-23} \mathrm{~J} / \mathrm{K}$ is the Boltzmann constant; the temperature of the interferometer is assumed to be close to the reference temperature $T_{0}=290 \mathrm{~K}$ at which $F_{a}$ is defined. It is worth pointing out that if the DUT noise is independent of the carrier power $P_{o}$, as it happens for the additive noise, $S_{\varphi 0}$ is decreased by increasing $P_{o}$.

The microwave amplifier is also a potential source of flicker noise because of nonlinear phenomena that up-convert the dc bias flicker, as shown in [6] and [7]. Hence, linearity must be ensured by keeping the residual carrier power at the amplifier input as low as possible; a carrier suppression of $60-80 \mathrm{~dB}$ 


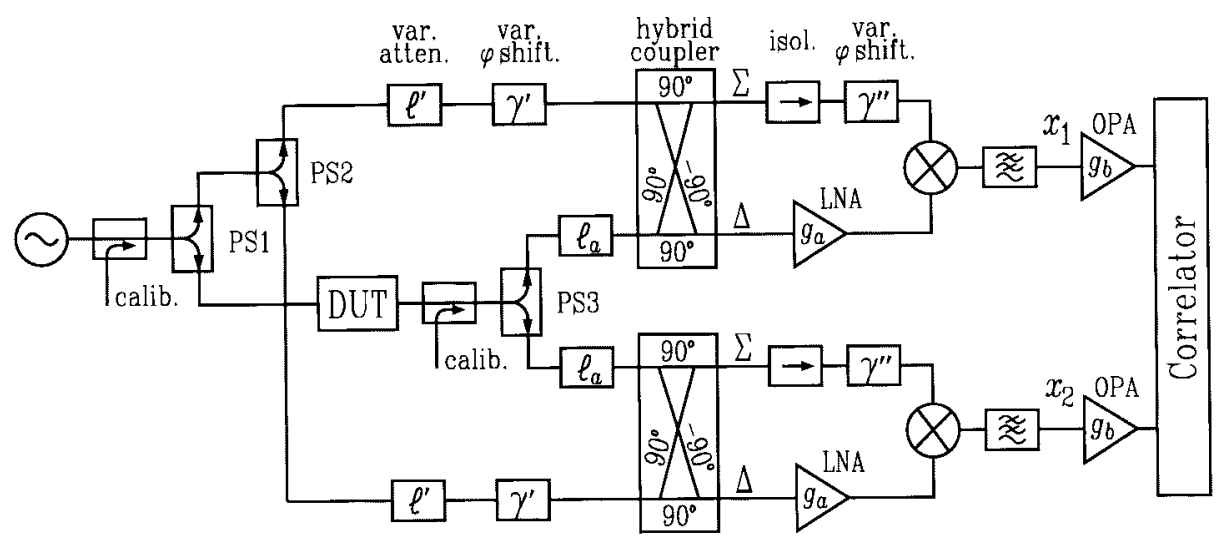

Fig. 1. Block diagram of the double interferometer.

can be necessary, depending on the implementation and $P_{\circ}$. Moreover, the phase shifters $\gamma^{\prime}$ and the attenuators $\ell^{\prime}$ can generate additional white and flicker PM noise.

Setting $\gamma^{\prime \prime}=0^{\circ}, x(t)$ is proportional to the instantaneous value $\alpha(t)$ of the relative amplitude noise. The above formulae (1) and (2) still hold, replacing $K_{\varphi}$ and $S_{\varphi 0}$ with $K_{\alpha}$ and $S_{\alpha 0}$, whose meanings are obvious.

We can now consider the whole scheme of Fig. 1, which allows the DUT noise to be measured through the cross PSD $S_{x_{1} x_{2}}(f)$ of the two interferometer outputs. Let us define $a, b$, and $c$ the noise processes taking place in the upper arm only, in the lower arm only, and in the common path, respectively. Accordingly, the output PSD's are $S_{x_{1}}=S_{a}+S_{c}$ and $S_{x_{2}}=S_{b}+S_{c}$. Provided that the detection phases $\gamma^{\prime \prime}$ are set equal, only the contribution of $c$ remains in the average product $\left\langle S_{x_{1}} S_{x_{2}}\right\rangle$, while all the cross terms are expected to approach zero as $1 / \sqrt{n}$, where $n$ is the number of measures, under the assumption that $a, b$, and $c$ are independent. So, we can measure $S_{c}$ by means of a digital correlator, and consequently, get $S_{\varphi}$ or $S_{\alpha}$, depending on $\gamma^{\prime \prime}$.

To sum up, all the sources of noise that are independent in the two arms are rejected by correlation. This applies to the most relevant ones, i.e., those located in the amplifiers, in the variable attenuators, and in the variable phase shifters. So, comparing our scheme to the single interferometer, a lower noise floor is expected or more noisy components can be adopted. The latter makes feasible an automatically tuned version of the double interferometer, which would require electrically controlled attenuators and phase shifters, generally more noisy than the manually adjustable ones.

\section{IMPLEMENTATION AND RESULTS}

We have built two NMS prototypes, one for the microwave band and one for VHF, described below.

The microwave NMS, shown in Fig. 2, operates at the carrier frequency $\nu_{c}=7.3 \mathrm{GHz}$, chosen because of the availability of all the components. The amplifier consists of two cascaded modules, showing an overall gain of about 45 $\mathrm{dB}$, a noise figure $F_{a}=2 \mathrm{~dB}$, a maximum power $P_{m}=18$ $\mathrm{dBm}(1 \mathrm{~dB}$ compression), and a bandwidth $B=250 \mathrm{MHz}$. This relatively narrow band is needed to ensure that the noise

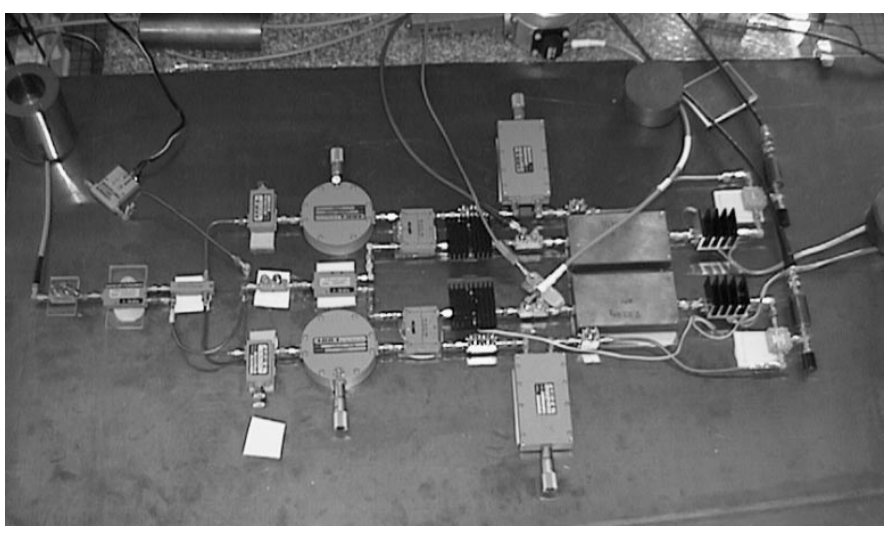

Fig. 2. Microwave implementation of the double interferometer.

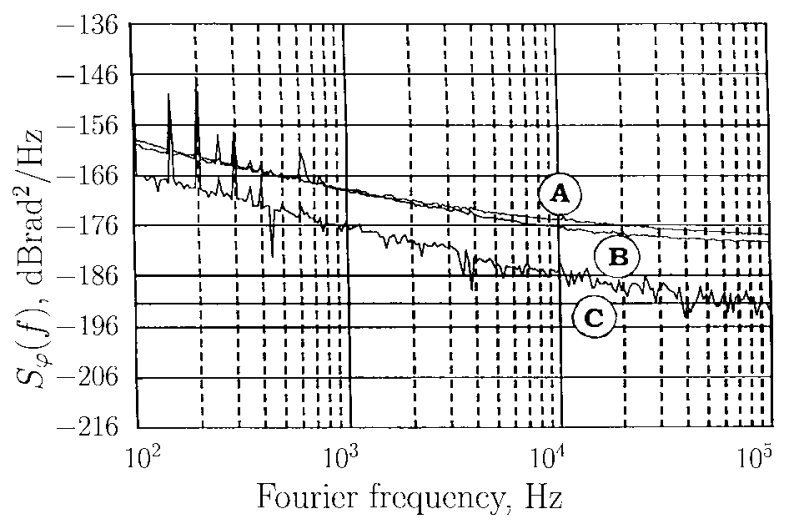

Fig. 3. Instrument noise of the microwave double interferometer. Curves A and B: single arm noise, and curve C: cross correlation.

power is sufficiently low to keep the amplifier in its fully linear region, thus preventing it to flicker. Operating with a DUT output power $P_{o}=15 \mathrm{dBm}, K_{\varphi}$ turns out to be $36 \mathrm{~dB} \mathrm{~V} / \mathrm{rad}^{2}$; the overall gain is $56 \mathrm{~dB} \mathrm{~V}^{2} / \mathrm{rad}^{2}$, including the amplifiers inserted between the mixers and the correlator $\left(g_{b}=20 \mathrm{~dB}\right)$. A directional coupler, inserted between the two stages, allows the monitoring of the residual carrier. Some ferrite isolators are inserted at the $\Sigma$ output of the hybrids to prevent oscillation or measurement alteration due the unwanted feedback through the hybrid, which shows poor isolation, of the order of $20 \mathrm{~dB}$. 


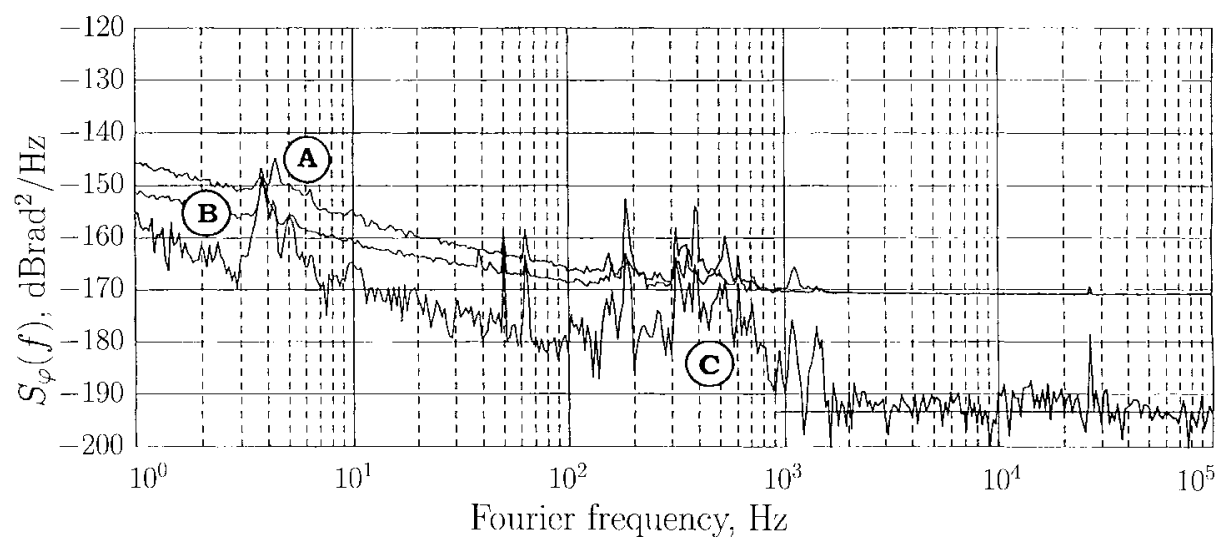

Fig. 4. Instrument noise of the VHF double interferometer. Curves A and B: single arm noise, and curve C: cross correlation.

A carrier suppression of -80 to $-90 \mathrm{~dB}$, stable for $30 \mathrm{~min}$, can be achieved. After observing that the source is critical because of its noise, we adopted the best available one, i.e., a cryogenic sapphire whispering gallery oscillator [8], which exhibits a white noise of $-180 \mathrm{~dB} \mathrm{rad}^{2} / \mathrm{Hz}$ and an estimated flicker noise of $-94 \mathrm{~dB} \mathrm{rad} / \mathrm{Hz}$ at $100 \mathrm{~Hz}$.

The VHF prototype, which operates at $\nu_{c}=100 \mathrm{MHz}$, is similar to the microwave one, but it was able to be constructed after solving certain additional problems, mainly due to the variable phase shifters and to harmonic distortion. The latter turns into a relevant difficulty because most VHF components show a bandwidth of (2-3) decades, while the carrier suppression mechanism is effective only at $\nu=\nu_{c}$ and the unwanted harmonics could be stronger than the residual fundamental. The carrier suppression of this NMS is about (65-70) $\mathrm{dB}$, limited by the variable attenuators, which are based on a potentiometer. In fact, these attenuators seem to be less stable than those based on a movable absorbing surface; the latter type, unfortunately, is not available for the VHF. On the other hand, because the driving oscillator is less critical than in the other band, we adopted a commercial device [9], which shows a white noise floor of $-157 \mathrm{~dB} \mathrm{rad}^{2} / \mathrm{Hz}$ and a flicker noise of $-127 \mathrm{~dB} \mathrm{rad} / \mathrm{Hz}$ at $100 \mathrm{~Hz}$. Operating with a DUT output power $P_{o}=8 \mathrm{dBm}, K_{\varphi}$ turns out to be 24 $\mathrm{dB} \mathrm{V}^{2} / \mathrm{rad}^{2}$, not including the amplifiers $\left(g_{b}=20 \mathrm{~dB}\right)$ at the output of the mixers. As design strategies and implementations are close to those of the single interferometers operating in the same bands, further details can be found in [10]. Because we are mainly interested in the sensitivity of our NMS's, we did all the noise measurements with a short cable replacing the DUT.

The microwave NMS was calibrated by means of a directional coupler inserted in the DUT path. After injecting an amplitude modulation through this coupler, we set $\gamma_{1}^{\prime \prime}$ and $\gamma_{2}^{\prime \prime}$ to null $x_{1}$ and $x_{2}$, the latter being monitored by a lock-in amplifier; in this condition, both the arms of the NMS detect the PM noise. Then, the gain $K_{\varphi}$ was measured by injecting a sideband of known amplitude in the coupler and measuring the amplitude of $x_{1}$ and $x_{2}$. After calibrating, the coupler input port was connected to a matched termination. The VHF NMS was calibrated by means of a varactor phase modulator, explicitly built for this purpose, inserted in the DUT path. $\gamma_{1}^{\prime \prime}$ and $\gamma_{2}^{\prime \prime}$ were set for the null of $x_{1}$ and $x_{2}$, monitored with the lock-in. Then, by inspecting with a network analyzer, $\gamma_{1}^{\prime \prime}$ and $\gamma_{2}^{\prime \prime}$ were increased by $90^{\circ}$. Finally, the varactor of the phase modulator was replaced by a less noisy variable capacitor, set to the same value.

The results of noise measurement for the microwave NMS are shown in Fig. 3. Curves A and B are the $S_{\varphi 1}(f)$ and $S_{\varphi 2}(f)$ of the two individual interferometers, while curve $\mathrm{C}$ is the cross PSD, which represents the sensitivity of the NMS. Unfortunately, the asymptotic values in the rightmost part of the figure can only be inferred because the flicker is not negligible even at $f=100 \mathrm{kHz}$, which is the maximum input frequency for the available correlator. Curves $\mathrm{A}$ and B approach values close to $-180 \mathrm{~dB} \mathrm{rad}^{2} / \mathrm{Hz}$, which is the noise floor predicted by (2) evaluated for the described implementation. At the lowest frequencies, below $10 \mathrm{kHz}$, the NMS noise (curve $C$ ) is of the flicker type, showing a value of $-167 \mathrm{~dB} \mathrm{rad} 2 / \mathrm{Hz}$ at $f=100 \mathrm{~Hz}$. Despite the use of the cryogenic oscillator, this flicker is probably due to the noise of the source instead of the NMS limit. In fact, we measured a source noise rejection of about $75 \mathrm{~dB}$, and the measured flicker is close to the oscillator noise attenuated by this rejection ratio. The white noise of the instrument, which can only be inferred in the rightmost part of Fig. 3, is approximately $-192 \mathrm{~dB}$ $\mathrm{rad}^{2} / \mathrm{Hz}$.

Similar measurements, made with the VHF NMS, yield the results shown in Fig. 4, where curves A, B, and C have the same meaning as before. The white noise of the individual arms (curves A and B) is $-172 \mathrm{~dB} \mathrm{rad} / \mathrm{Hz}$, close to the value predicted by (2). At the lowest frequencies, $f<1 \mathrm{kHz}$, the NMS noise (curve $C$ ) is of the flicker type, showing a value of $-171 \mathrm{~dB} \mathrm{rad} / \mathrm{Hz}$ at $f=10 \mathrm{~Hz}$. In addition, the effect of acoustic vibrations is dominant in the region between $200 \mathrm{~Hz}$ and $2 \mathrm{kHz}$. The white noise floor is $-194 \mathrm{~dB} \mathrm{rad}^{2} / \mathrm{Hz}$.

We wish to stress that no signal processing was done to mask any disturbance-like those due to acoustic vibrations, electromagnetic pollution, or the residual of the mains-before reporting the results shown in Figs. 3 and 4. The noise measurements were done placing the NMS's on an antivibrating table, of the same type of those commonly used for optics, 
without using a shielded chamber. The above results were able to be obtained after a refinement of the mechanical assembly and a careful choice of the path of cables, ground, and power supply.

\section{Discussion OF THE RESUlts AND CONCLUSION}

Basically, the double interferometer noise floor is determined by the noise sources shared by the two arms. The most relevant of these sources are the power splitter internal resistors, whose noise $k_{B} T_{0}$ is equally divided into the two outputs. In fact, the power splitters PS1-PS3, which are of the reactive type, are actually four-port $3 \mathrm{~dB}$ hybrid couplers with one input connected to a resistive termination $\left(R_{1}, R_{2}\right.$, and $R_{3}$, respectively). Let us first consider $R_{1}$. Because of the phase relationships shown in Fig. 1 , the noise $k_{B} T_{0}$ is equally divided into the two LNA inputs. Taking into account the losses, noise contributions $k_{B} T_{0} /\left(2 \ell_{s}^{2} \ell_{a} \ell_{h}\right)$ are expected at the two LNA inputs, fully correlated because they come from a single source. For similar reasons, $R_{2}$ and $R_{3}$ yield correlated noise. Summing the noise power due to $R_{1}, R_{2}$, and $R_{3}$ at the LNA inputs, we get a fully correlated noise $k_{B} T_{0} /\left(\ell_{s}^{2} \ell_{a} \ell_{h}\right)$; the complement to $k_{B} T_{0}$, due to the individual arm losses, is uncorrelated. Taking into account the NMS gain (1), the expected noise floor of the double interferometer is

$$
S_{\varphi \mathrm{thdi}} \geq \frac{4 k_{B} T_{0}}{P_{o}}
$$

where the equality holds in the case of minimum losses. It should be observed that the lower bound given by (3) is $6 \mathrm{~dB}$ higher than the thermal noise limit $S_{\varphi \mathrm{th} 0}=k_{B} T_{0} / P_{o}$. This is due to the power splitter PS3 and the hybrids, which limit to $1 / 4$ the fraction of the DUT output noise that reaches the input of each LNA.

Because the transmission coefficients from the power splitter internal resistors $\left(R_{1}, R_{2}\right.$, and $\left.R_{3}\right)$ to the LNA inputs are considered relevant to the comprehension of the noise floor, we used externally terminated 4-port hybrids as power splitters in the VHF NMS, and we measured these coefficients by means of a network analyzer. The transmission coefficients turn out to be $-6 \mathrm{~dB}$ from the $R_{1}$ port to the LNA inputs, and -8.5 $\mathrm{dB}$ from the $R_{2}$ and $R_{3}$ ports to the LNA inputs, in a close agreement to the expected values. The latter are based on the nominal losses $\ell_{s}=0.8 \mathrm{~dB}, \ell_{a}=1 \mathrm{~dB}$, and $\ell_{h}=0.8 \mathrm{~dB}$ of the available devices.

Although the resistances $R_{1}, R_{2}$, and $R_{3}$ seem to give correlated contribution to the two arms of the NMS, the measured noise floor $S_{\varphi 0}$ is lower than that predicted by (3). In fact, the microwave NMS shows a noise floor of $-192 \mathrm{~dB}$ $\mathrm{rad}^{2} / \mathrm{Hz}$, while (3) yields $-183 \mathrm{~dB} \mathrm{rad} / \mathrm{Hz}$ for $P_{o}=15 \mathrm{dBm}$. Moreover, the VHF prototype shows a noise floor of -194 $\mathrm{dB} \mathrm{rad}^{2} / \mathrm{Hz}$, which is $18 \mathrm{~dB}$ lower than the value predicted by (3) for $P=8 \mathrm{dBm}$. A theoretical explanation for the above result is still under development. Anyway, our results should be compared to those reported in [11], where another correlation-based NMS is described, showing an exceptionally low noise floor, of the order of $-200 \mathrm{~dB} \mathrm{rad}^{2} / \mathrm{Hz}$ at $\nu_{c}=5$ $\mathrm{MHz}$. That scheme is different from ours, mainly because of the absence of the carrier suppression mechanism and because the mixers are used as phase-to-voltage converters, saturated at both inputs. Although only a brief system description is given in [11], and the presence of thermal noise sources common to the two arms due to the power splitter internal resistors is not mentioned, the reported noise floor is some $10 \mathrm{~dB}$ lower than $k_{B} T_{0} / P_{o}$.

To conclude, the double interferometer allows noise measurements with a lower instrument noise floor as compared to single interferometer systems. Our prototypes, although not optimized, prove the feasibility and the benefits of the proposed scheme in a wide frequency range. Further work is in progress, to give an adequate theoretical explanation for the reported results.

\section{REFERENCES}

[1] K. H. Sann, "The measurement of near-carrier noise in microwave amplifiers," IEEE Trans. Microwave Theory Tech., vol. MTT-16, pp. 761-766, Sept. 1968.

[2] E. N. Ivanov, M. E. Tobar, and R. A. Woode, "A study of noise phenomena in microwave components using an advanced noise measurement system," IEEE Trans. Ultrason., Ferroelect., Freq. Contr., vol. 44, pp. 161-163, Jan. 1997.

[3] D. Fest, J. Groslambert, and J. J. Gagnepain, "Individual characterization of an oscillator by means of cross-correlation or cross covariance method," IEEE Trans. Instrum. Meas., vol. IM-32, pp. 447-450, Sept. 1983.

[4] E. Rubiola, V. Giordano, and J. Groslambert, "Double correlating interferometer scheme to measure PM and AM noise," Electron. Lett., vol. 34, Jan. 1998.

[5] _ "VHF and microwave interferometric PM and AM noise measurements," in Proc. 12th Eur. Frequency Time Forum, Warszawa, Poland, Mar. 10-12, 1998.

[6] V. N. Kuleshov and T. I. Boldyreva, " $1 / f$ AM and PM noise in bipolar transistor amplifiers: Sources, ways of influence, techniques of reduction," in Proc. 51st Frequency Control Symp., Orlando, FL, May 28-30, 1997, pp. 446-455.

[7] F. L. Walls, E. S. Ferre-Pikal, and S. R. Jefferts, "Origin of $1 / f$ PM and AM noise in bipolar junction transistor amplifiers," IEEE Trans. on Ultrason., Ferroelect., Freq. Contr., vol. 44, pp. 326-334, Mar. 1997.

[8] O. Di Monaco, "Résonateurs saphyr micro-onde cryogéniques à très grand coefficient de surtension pour applications métrologiques," Ph.D. dissertation, Univ. de Franche Comté, Besançon, France, June 1997.

[9] "Pulsar-H," AR Electronique, France, 1997.

[10] E. Rubiola, V. Giordano, and J. Groslambert, "VHF and microwave interferometric PM and AM noise measurements," Rev. Sci. Instrum., Jan. 1999.

[11] F. L. Walls, S. R. Stain, J. E. Gray, and D. J. Glaze, "Design considerations in state-of-the-art signal processing and phase noise measurement systems," in Proc. 30th Frequency Control Symp., Atlantic City, NJ, June 2-4, 1976, pp. 269-274.

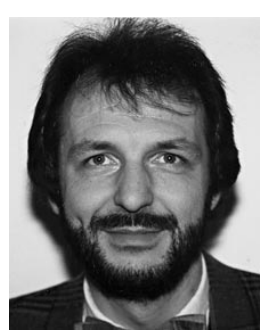

Enrico Rubiola was born in Torino, Italy, in 1957. $\mathrm{He}$ received the Laurea degree in electronic engineering in 1983, and the Ph.D. degree in metrology in 1989, both from the Politecnico di Torino.

Since 1990, he has been a Permanent Researcher, Dipartimento di Elettronica, Politecnico di Torino, where he is also in charge of two courses on measurements for telecommunications. From 1983 to 1992 , he worked on navigation systems and time and frequency comparison. Since 1990, he has been interested in frequency synthesis, precision electronics, and precision phase noise measurements. The latter subject has recently been extended to high spectral purity oscillators and noise metrology. He cooperates with the Laboratoire de Physique and Métrologie des Oscillateurs, Besançon, France, where he has spent time as an Invited Researcher. 


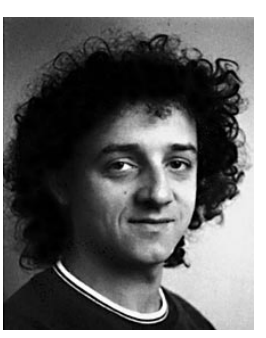

Vincent Giordano was born in Besançon, France, in 1962. He received the engineer degree in 1984 from the Ecole Supérieure de Mécanique et des Microtechniques, Besançon, and the Ph.D. degree in physical sciences in 1987 from the Paris XI University, Orsay, France.

From 1984 to 1993 , he was a Researcher on the Permanent Staff of the Laboratoire de l'Horloge Atomique, Orsay, where he worked on a laser diode optically pumped cesium beam frequency standard. In 1993, he joined the Laboratoire de Physique et de Métrologie des Oscillateurs, Besançon, where he is the head of the Microwave Metrology team. Presently, his main area of interest is the study of high spectral purity microwave oscillators and the high sensibility phase noise measurement systems.

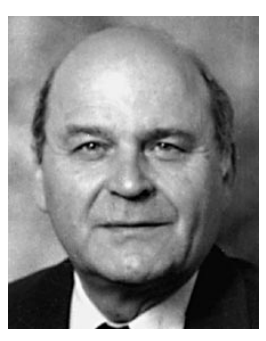

Jacques Groslambert was born in Besançon, France, in 1938. He received the Diplóme d'Ingénieur INSA in electrical engineering from the Institut National des Sciences Appliquées, Lyon, France, in 1961, and the Dr. Ing. degree from the Université de Franche Comté, Besançon, in 1969.

Since 1963, he has been working on multiplier chains, frequency synthesis, and noise measurement systems with the Laboratoire de Physique et de Métrologie des Oscillateurs, Besançon. 